\title{
User preferences for multi-device context-aware feedback in a digital coaching system
}

\author{
Randy Klaassen • Rieks op den Akker • \\ Tine Lavrysen • Susan van Wissen
}

Received: 30 March 2012 / Accepted: 12 April 2013 / Published online: 5 May 2013

(C) The Author(s) 2013. This article is published with open access at Springerlink.com

\begin{abstract}
We present the development of the user interfaces of a multi-device digital coaching service that provides tailored feedback to users concerning their physical activity level and medication intake. We present the outcomes of a survey study of user preferences regarding the situation, device and timing of feedback they receive from their personal attentive digital coach. There are clear preferences among the subjects for different types of messages on different devices. Results were implemented in a first prototype. We present the results of a user evaluations with a real version of the digital health coach and we compare them with the results of the survey study.
\end{abstract}

Keywords Persuasive technology - User preferences . User interface design · User evaluations

This work was funded by the European Commission, within the framework of the ARTEMIS JU SP8 SMARCOS project 100249 (http://www.smarcos-project.eu).

R. Klaassen $(\bowtie) \cdot$ R. op den Akker

Human Media Interaction, University of Twente,

7500 AE Enschede, The Netherlands

e-mail: r.klaassen@utwente.nl

R. op den Akker

e-mail: h.j.a.opdenakker@utwente.nl

T. Lavrysen

Human Interface Group, 2800 Mechelen, Belgium

e-mail: tine.lavrysen@higroup.com

URL: http://www.higroup.com

S. van Wissen

Evalan B.V., 1018 AV Amsterdam, The Netherlands

e-mail: susan.vanwissen@evalan.com

URL: http://www.evalan.com

\section{Introduction}

In the EU Artemis project Smarcos that aims at developing methods and model based tools for inter-usable user interfaces, we developed a personal digital health coach that supports users in attaining a healthy lifestyle by giving timely, context-aware feedback about daily activities through a range of interconnected devices $[9,15]$. After an analysis of potential target groups we have chosen diabetes type II patients and office workers. A growing number of people suffers from diabetes type II. Diabetes is a complex disorder that requires constant attention to diet, exercise, glucose monitoring, and medication to achieve good glycemic control. Reviews confirm that many patients for whom diabetes medication was prescribed were poor compliers with treatment [5]. Electronic monitoring systems however have shown to be useful in improving adherence for individual patients [19]. Project partner Evalan was involved in the Dutch NIVEL research with the Real Time Medication Monitoring system with diabetics patients. This research showed that awareness of being monitored and receiving SMS reminders has a positive effect on medication adherence for this group. Office workers were chosen as a second target group because of their sedentary lifestyle which forms a serious threat for becoming obese and for developing chronic diseases such as diabetes at a later age (hence the WHO Global Strategy on Diet, Physical Activity and Health). The office workers get feedback on their activity level and food intake, while for diabetics medication intake and glucose values are also taken into account. They get personalized advice and tips, they can request an overview of results from the past or learn more about how their life style could influence their health. Many personal coaching systems exist nowadays, such as SitCoach, suggesting sitting breaks to office workers [6]. They found their way in health care since one of the focus areas in the treatment of various 
chronic diseases such as Chronic Obstructive Pulmonary Disease (COPD), Chronic Low Back Pain (CLBP), and Obesity is to balance or increase overall activity in everyday life [14]. Most of these systems provide feedback through one type of (mobile) device. Behavior change support systems [13] that are able to intervene with several contact points are expected to be more effective in stimulating health behavior changes than those that use a single contact point [12]. With the existence of behavior change support systems that can provide the user with information though different output devices in different contexts the issue is raised what users prefer [11].

First, we wanted to see in which situations users would like to receive what kind of feedback on which device. Then, we wanted to know whether user like to have messages presented by means of a text message or by a spoken presentation using an animated virtual human. In this paper, the results of two online questionnaires and two user evaluations are presented, for each of the two target groups. The questionnaire was also used to get an idea of how participants perceive the presented concept. The results of this user survey were used in the implementation of the first prototype of the Smarcos digital coaching system. The prototype was used in the first user evaluations.

This paper is organized as follows. In the next section we give a short description of the Smarcos digital coaching system, and how we developed this in a user centered way. In Sect. 3 we describe the method and results of the questionnaires for both target groups. In Sect. 4 we present the results of two small user evaluations studies with the first Smarcos prototype system. In one user evaluation five diabetes type II patients used the prototype system for 1 week. In the second user evaluation nine office workers participated for 2 weeks. In this user evaluation we compared two different ways of presenting the feedback. One way was by a simple text message, while in the other version we used an embodied conversational agent to present the feedback using verbal and non-verbal behaviour. We present main conclusions in Sect. 5 and we discuss ongoing and future research. ${ }^{1}$

\section{A multi-device digital personal coaching system}

Creating meaningful and effective attentive personal systems implies that these systems provide support taking the context of the user into account, and empower users to take control of their lifestyle. With the ultimate goal to support chronic patients in their daily life, feedback is used to empower users to adopt a certain (more healthy) target behaviour. In order to provide feedback at the right time and to use the right modality it is necessary to take into account the context of

\footnotetext{
1 Video presentations of the Smarcos coaching system can be found on YouTube: http://youtu.be/am4fMcz1qrs.
}

the user. Therefore feedback models define the interactions between the Smarcos digital coaching system and the user.

To capture the requirements, two context mapping studies have been conducted. Context mapping is a procedure for conducting in-depth research with users. The objective of this procedure is to gain knowledge about the use of products from implied and implicit information that is provided by prospective users during an intensive process [17]. Through active participation by users and stakeholders during the design process, it informs and inspires design teams and ensures a good fit between the design and the use of a product. These qualitative studies consisted of a diary booklet with exercises and a semi-structured interview. Over a period of 3 to 5 days, users were probed to describe their daily activities (like lunch behaviour and physical activities) and reflect on their behaviours relevant for the Smarcos system.

The study provided insights in the daily behaviour of the users, awareness of the importance of healthful behaviour and their barriers towards a desired behaviour. The requirements collected with this approach were further refined through a role playing exercise during a workshop in Amsterdam in which the majority of the Smarcos team members participated. The list of requirements that were identified included the following:

- The system needs to learn the specific Diabetes condition of the user.

- The system needs to know the relevant context information

- The system needs to teach and/or assist the user about the desired behaviour

- The system needs to be geared towards motivating the user

The feedback models used in the Smarcos digital coaching system are determined by the available sensors, input devices and devices for feedback. A feedback model describes the entire loop from capturing user behaviour, interpretation of inputs and reasoning about desired feedback. For a graphical representation of the basic elements that comprise a feedback model, see Fig. 1. Figure 2 shows the feedback model for the diabetes use case.

Awareness of one's own behaviour and the consequences for one's health is one of the key processes for health behavior change (e.g., $[3,16])$. To induce improvements in the selected behavioural domains, we focus on creating awareness of users' own behaviour and on the personal consequences of their behaviour in terms of blood glucose levels, physical activity and medication intake. To create awareness, simple and meaningful overviews of the users' behaviour and the outcome variables will be provided to them at opportune moments. Moreover, users can interact with the system to 
learn the effects of certain behaviour on their health. In this way the patient can learn to manage for example blood glucose levels by compensating the effects of certain behaviours (e.g. over-eating) through the engagement in other behaviours (e.g. physical activity). To induce behaviour change, we focus on self-regulation strategies that are offered in a tailored manner. Depending on the users' level of knowledge, the stage-of-change they are in and their psychological characteristics, the system can provide informational, motivational or behavioural support to promote behaviour change towards a healthier lifestyle. We distinguish four different contexts: at home, at work, outside and on the go. In these different contexts, different devices are available for presenting feedback. Some devices can use different modalities for presenting feedback. Depending on the user's context, the system should select the right device and modality to present the feedback on.
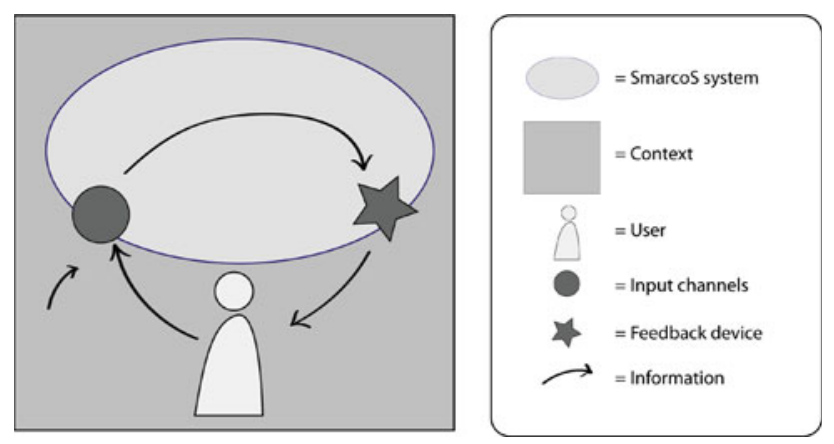

Fig. 1 Graphical representation of basic elements for the Smarcos feedback model

\subsection{The reference architecture}

Figure 3 shows the reference architecture of the Smarcos digital coaching system prototype. Figure 3 shows the different in- and output devices, two different contexts of the user and the Smarcos cloud services. The first prototype makes use of two output devices: a smartphone (Android or iOS) and a desktop computer or laptop, and three input devices: a pill dispenser, an activity monitor and GPS location (provided by the smartphone). All input and output devices are connected to the Smarcos cloud. User profiles and preferences, contextual information and sensors data is uploaded to the cloud and stored in a central database. The orchestrator continuously keeps track of all user data and contains coaching rules. When the orchestrator receives a trigger it starts to evaluate the coaching rules. When one of the rules is true, it will select a suitable message from the coaching content database and send the message to the user through one of the available output devices and through one of the available modalities.

The feedback coach has to decide what the best opportunity is for giving specific feedback. In order to get a general view on when users prefer to receive feedback and on what device, we carried out the user survey reported in the following sections. Default settings will be based on this general knowledge and can be personalized per user.

\section{Online user evaluation of the Smarcos prototype}

Two questionnaires were online from September to November 2011. The office worker questionnaire was spread

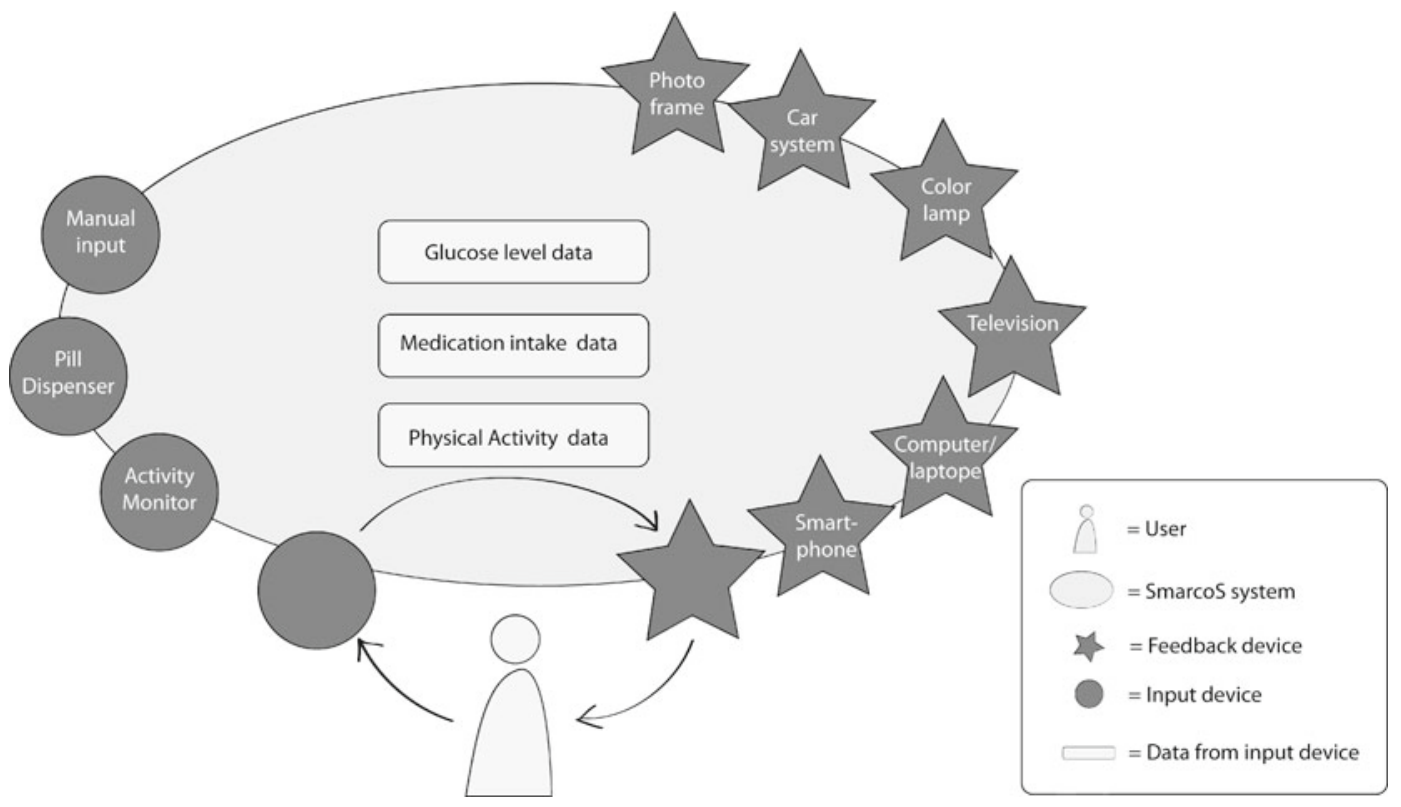

Fig. 2 Full model for the diabetes use case 


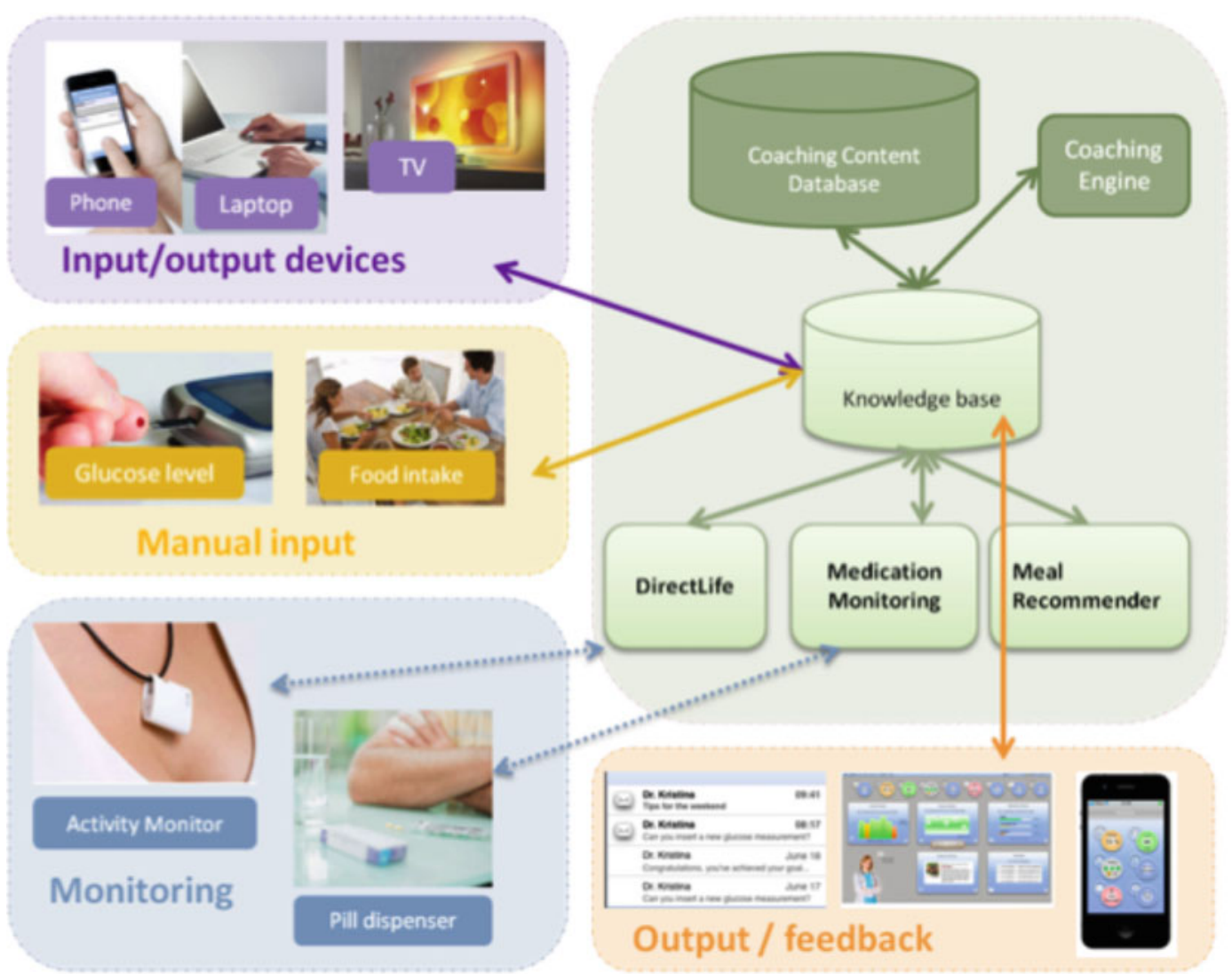

Fig. 3 Overall architecture of the coaching system

amongst project partners, who spread it amongst their colleagues. Diabetics were found through personal contacts with a doctor, and through online forums.

\subsection{Goal of the questionnaire}

User were asked to imagine different scenarios in which they could get feedback. Different aspects of the Smarcos coach prototype were tested.

1. Scenario-based questions: based on the description of a specific situation (see later), the users were asked:

(a) Which types of messages are suitable to be sent through which devices?

(b) In which situations is it acceptable to send feedback? What are situations they would like to get reminders/support?

2. General impression With the help of the word-pairs based on the AttrakDiff ${ }^{2}$ questionnaire, users were asked to rate the concept as described during the questionnaire.

\footnotetext{
${ }^{2}$ http://www.attrakdiff.de.
}

\subsection{Subjects demographics}

\subsubsection{Diabetes questionnaire participants}

Fifteen participants completed the diabetes questionnaire: 4 males, 11 females. 2 were younger than 25 years old, 7 between 25 and 50 years old, and 6 were older than 50 years old. The largest part of our diabetic participants were born in The Netherlands.

Eight participants were diagnosed with diabetes more than 3 years ago, 2 participants between 2 and 3 years ago, 2 participants between 1 and 2 years ago, and 2 participants less than 6 months ago.

\subsubsection{Office questionnaire participants}

Forty-nine participants completed the healthy office worker questionnaire: 26 males, 23 females. 2 were younger than 25 years old, 41 participants were between 25 and 50 years old, and 6 were older than 50 years old. Participants' countries of origin were among others: Belgium (17 participants), The Netherlands (15 participants) and Spain (8 participants).

The respondents to our questionnaire spent on average $7.5 \mathrm{~h}$ seated (in front of a computer) on an average working day. 


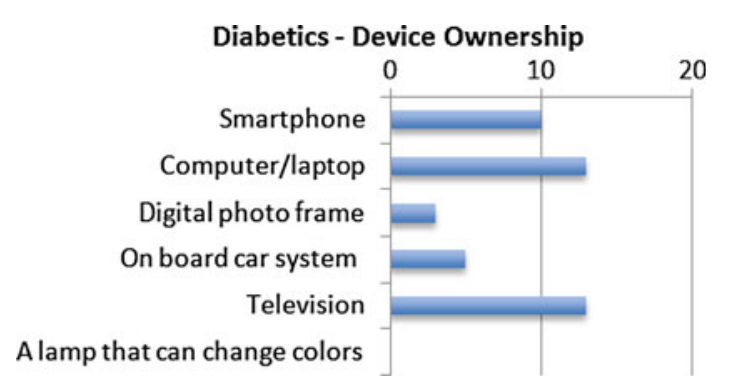

Fig. 4 Diabetics device ownership

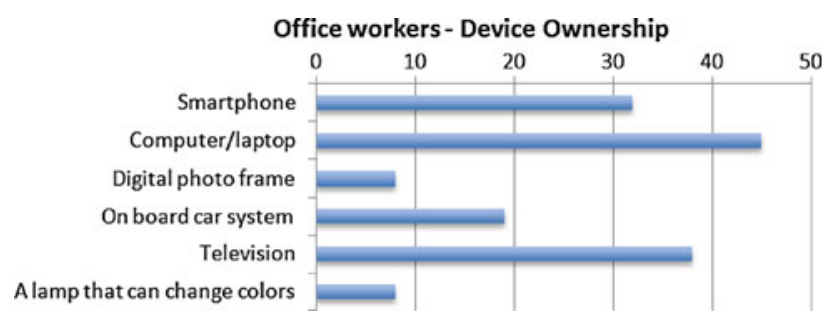

Fig. 5 Office workers device ownership

\subsubsection{Device ownership}

Most participants owned a computer/laptop, many owned a television and many owned a smartphone. About one in three owned an on-board car system (such as a navigation system) and less than one in five owned a digital photo frame. One in six participants owned a color-changing lamp, all of which were office workers. Figures 4 and 5 show the device ownership of both user groups.

\subsection{Results scenario-based questions}

Here we present the results of the scenario-based questions.

\subsubsection{Scenario-based questions}

Participants were asked to imagine different scenario in which they could get feedback. Then they were asked which type of messages would be suitable to be sent through which devices and in which scenarios it would be acceptable to receive specific messages.

\subsubsection{Which types of messages are suitable to be sent through which devices?}

The possible scenarios are partly different for diabetics and for office workers. Scenarios 3 and 5 were identical for both target groups.

Scenarios for Diabetics In this part of the questionnaire, users were presented with a specific situation combined with a specific message. They were then asked to indicate on which of the presented devices they would like to receive that mes- sage in that situation. Multiple devices could be selected. Afterwards, they were asked to pick a preferred device.

Scenarios and messages:

- Scenario 1: You are at home in a hurry to go to work. Message: A medicine reminder: 'Did you forget your medicine?'

- Scenario 2: You are at home cooking diner. Message: A medicine reminder: 'Did you forget your medicine?'

- Scenario 3: You are relaxing in front of the television. Message: An overview of your physical activity level of the last week. You can see how many calories you have burned per day during the last week.

- Scenario 4: You are still relaxing in front of the television. Message: Request for providing a new glucose measurement.

- Scenario 5: You are on working during a normal working day. Message: A request to set your personal physical activity goals for the coming time.

Device options: Smartphone, Computer/Laptop, Digital photo frame, Car system,Television and/or Color-changing lamp.

Responses from Diabetics The most popular devices for receiving messages are the smartphone and personal computer. Especially when in a hurry to go to work the smartphone is a device that most respondents liked to receive feedback on. When they are at home, the television is also a device users would like to get feedback on.

When the diabetic respondents are asked which device has their preference, the same top-3 is visible: 1 . Smartphone, 2. Computer/laptop, 3. Television. The other three devices (digital photo frame, on-board car system, and color-changing lamp) are not even mentioned once in the list of preferred devices (Figs. 6, 7).

Scenarios for Office Workers Similar questions were asked to the office workers, although they were presented with different specific situations combined with specific messages. Scenarios 3 and 5 were identical. The office respondents were also asked to indicate on which of the presented devices they would like to receive that message in that specific situation. Multiple devices could be selected. Afterwards, they were also asked to pick a preferred device.

Scenarios and messages:

- Scenario 1: You are at home in a hurry to go to work. Message: 'You have been sitting for a long time, time for a walk?'

- Scenario 2: You are at home cooking diner. Message: 'You have been sitting for a long time, time for a walk?'

- Scenario 3: You are relaxing in front of the television. Message: An overview of your physical activity level of 
Diabetics - On which of the devices would you like to receive this message?

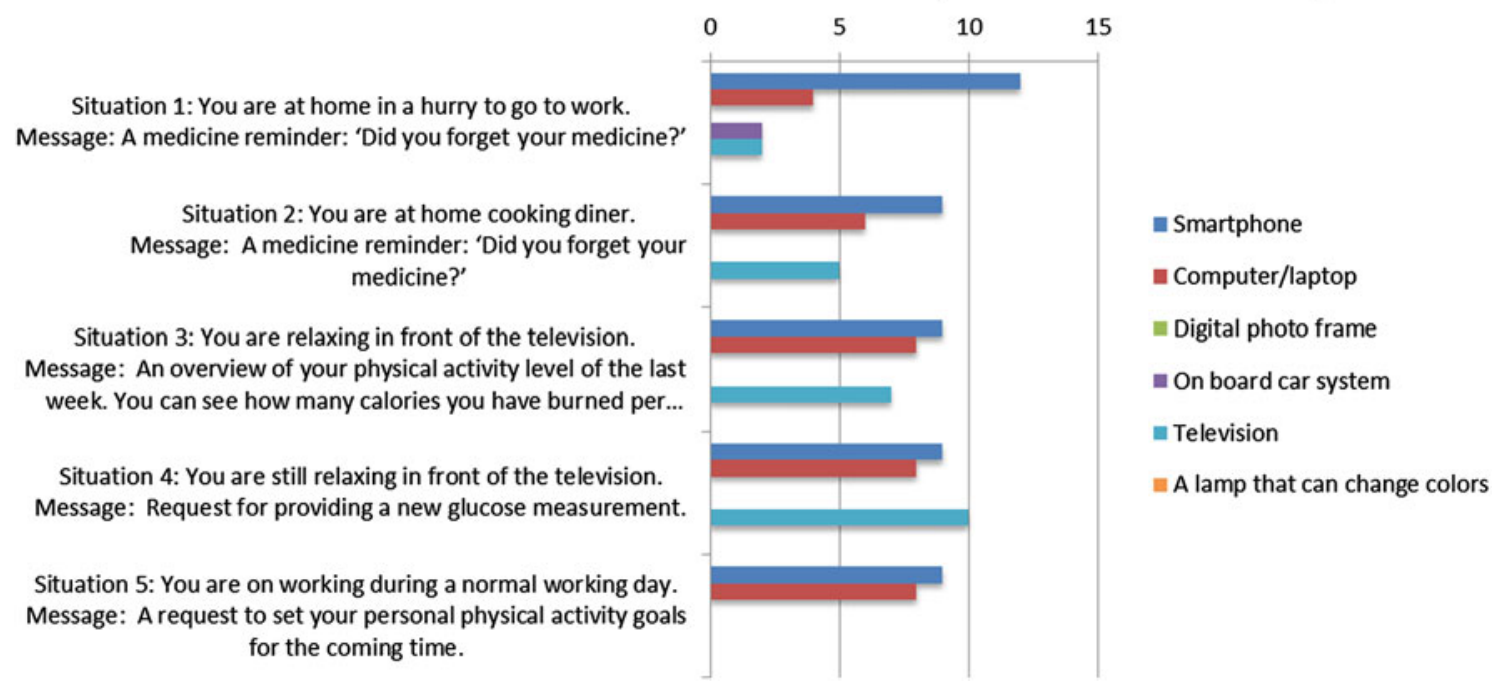

Fig. 6 Device preferences per scenario (diabetics)

\section{Diabetics - In which situation would you like to receive this message?}

Message 1: An overview of your progress towards your physical activity goal of that day.

Message 2: An explanation of the effect of physical activity on your health.

Message 3: A motivating text (e.g. "You are on the right track")

Message 4: Physical activity goal achievement: 'Congratulations you achieved you physical activity goal of the day."

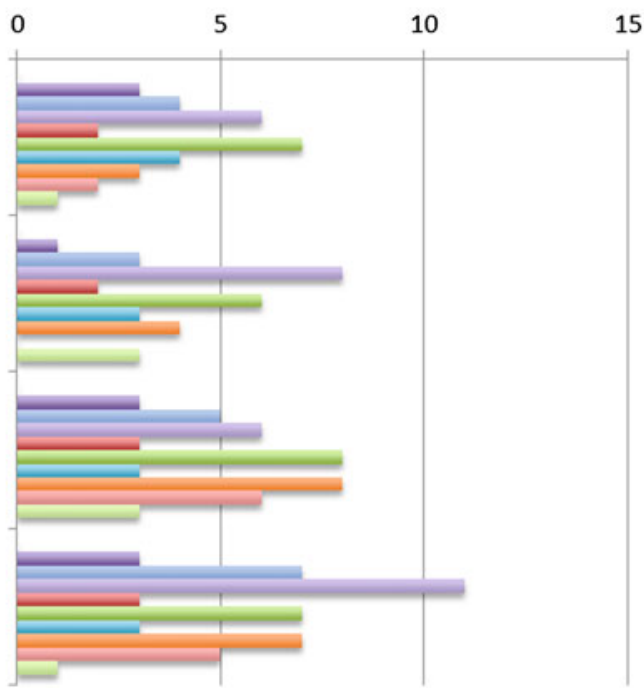

15

At home in a hurry
$=$ Cooking dinner
$=$ Having dinner
$=$ Relaxing in front of the television
$\equiv$ Working during a normal day
$=$ Having a (lunch) break
$=$ Travelling through public
transport
$=$ Walking outside
$\equiv$ Doing sports
None of the above

Fig. 7 Device preferences per scenario (office workers)

the last week. You can see how many calories you have burned per day during the last week.

- Scenario 4: You are still relaxing in front of the television. Message: Request for inserting a meal (telling the system what you have eaten today).

- Scenario 5: You at work during a normal working day. Message: A request to set your personal physical activity goals for the coming time.

Device options: Smartphone, Computer/Laptop, Digital photo frame, Car system, Television and/or Color-changing lamp.

Responses from Office Workers The most popular devices for receiving messages are the smartphone, personal computer, and television again. The computer/laptop is most popular when the user is in the office, and loses popularity when users are at home. There the television is a device users would like to get feedback on, next to the smartphone. The on-board car system got only a small amount of votes, but contrarily to the diabetic respondents, the color-changing lamp is seen as a device that some office workers would like to receive feedback on.

As for the preferred device in each situation, the computer/laptop is clearly the winning device when a request is sent to the user while he is in the office.

In the other situations, the preferences are more scattered. When in a hurry to go to work, a reminder for hav- 
Office workers - On which of the devices would you like to receive this message?

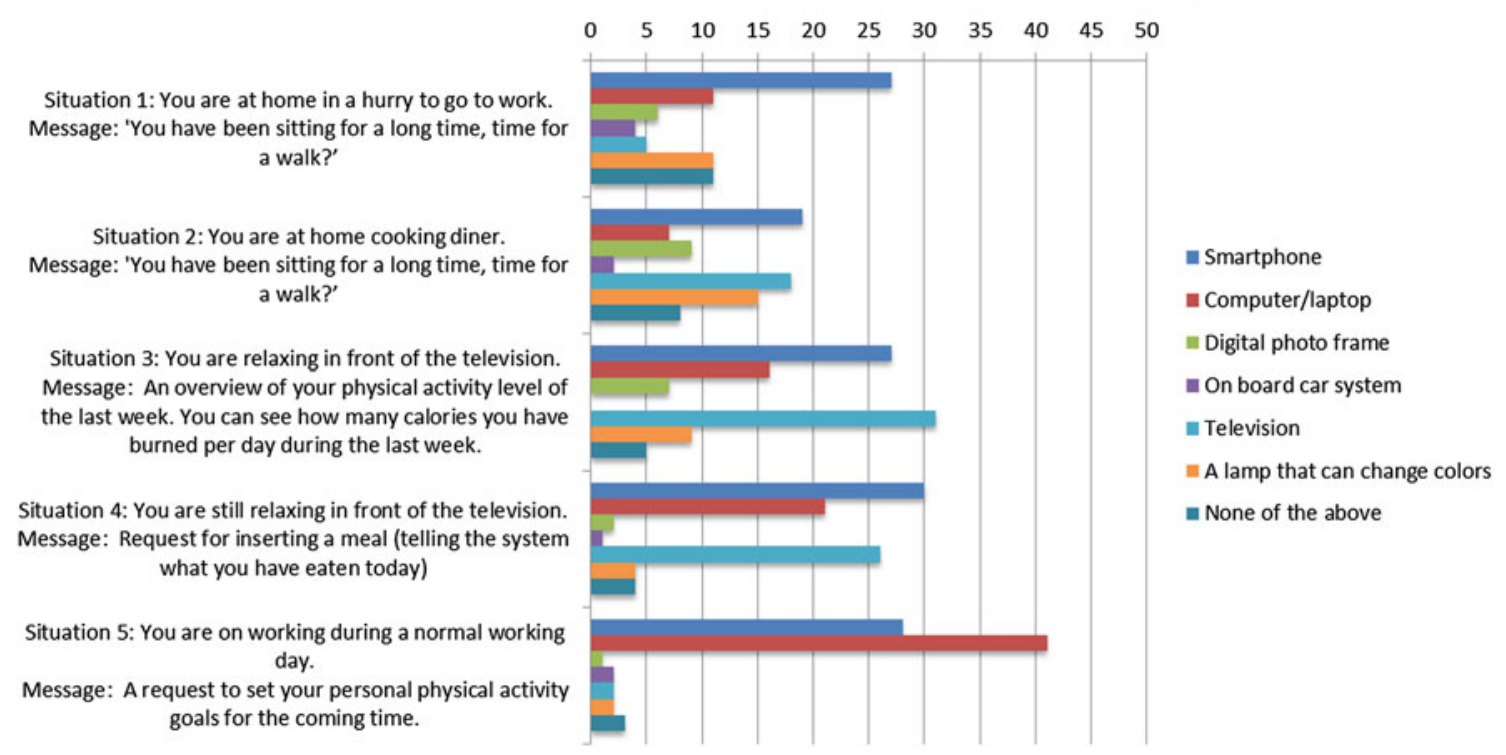

Fig. 8 Situation preferences for receiving messages (diabetics)

Office workers - In which situation would you like to receive this message?

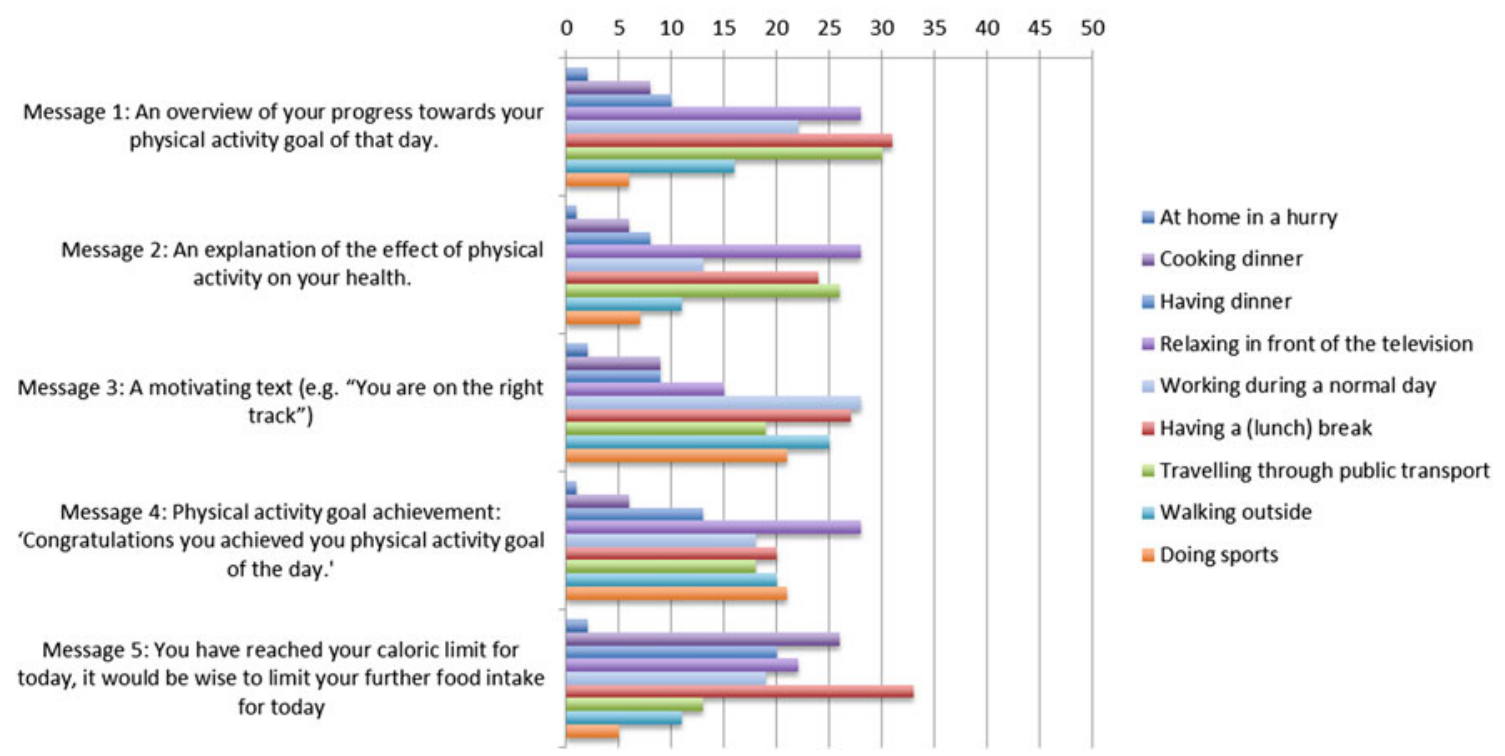

Fig. 9 Situation preferences for receiving messages (office workers)

ing a sitting break is preferably sent to the smartphone, but the computer and color-changing lamp also had some preference votes. While cooking, the smartphone and lamp are still popular, but the television also gained a lot of votes.

While sitting in front of the television, respondents are divided in people that prefer to receive messages on the TV itself, or people that prefer a message on their smartphone (Figs. 8, 9).

\subsubsection{In which situations is it acceptable to send feedback/reminders/support?}

Scenarios for Diabetics The same specific messages were described to the office workers, and the respondents were asked in which situations they would like to receive that message. Multiple situations could be selected. Afterwards, they were also asked which was their preferred moment of receiving that specific message. 
Messages:

- Message 1: An overview of your progress towards your physical activity goal of that day.

- Message 2: An explanation of the effect of physical activity on your health.

- Message 3: A motivating text (e.g. "You are on the right track").

- Message 4: Physical activity goal achievement: 'Congratulations you achieved you physical activity goal of the day.'

Scenario options:

- At home in a hurry

- Cooking dinner

- Having dinner

- Relaxing in front of the television

- Working during a normal day

- Having a (lunch) break

- Traveling through public transport

- Walking outside

- Doing sports

At the end of this series, the respondents were asked which messages they would like to receive, provided that they would only be sent at appropriate moments during the day. Multiple messages could be selected.

Message options:

- An overview of your physical activity level of the last week

- An overview of your medication adherence of the last week

- Progress towards achieving your physical activity goal of the day

- A request for providing a new glucose measurement

- A request to set your physical activity goal

- A message that you have achieved your physical activity goal

- A motivational text (e.g. "You are on the right track")

- A medicine reminder: "Did you forget to take your medicine?

- General tips on how to become more physically active

- An explanation of the effects of being physical active on your health

Responses from Diabetics The most popular situations for receiving different types of messages overall seem to be while relaxing in front of the television, and while having a (lunch) break. While walking outside or while doing sports, motivating and congratulatory messages are also welcomed by a large part of the respondents.

Of the messages they were presented with, the diabetic respondents did not want to receive any message while at home in a hurry. While cooking dinner, while working during a normal day and while travelling through public transport were all equally unpopular, with on average about 1 in 5 respondents who would like to receive the presented messages in that situation. Interestingly, almost half of the diabetic respondents would not mind getting a congratulatory message that they have achieved their daily activity target while having dinner.

When the diabetic respondents were asked which messages they would like to receive, provided that the timing was good, two-thirds would like to receive a message containing their physical activity level of the last week, and half of them wanted to see their progress towards their daily physical activity goal.

Less than half of the respondents indicated to welcome messages with their weekly medicine intake or requests for a new glucose measurement. Only about a third of the respondents indicated to wish to receive the other messages.

Scenarios for Office Workers Similar questions were posed for the timing of messages. Here specific messages were described, and the respondents are asked in which situations they would like to receive that message. One extra message about caloric intake was added for the office workers. Multiple situations could be selected. Afterwards, they were asked which was their preferred moment of receiving that specific message.

Messages are:

- Message 1: An overview of your progress towards your physical activity goal of that day.

- Message 2: An explanation of the effect of physical activity on your health."

- Message 3: A motivating text (e.g. "You are on the right track").

- Message 4: Physical activity goal achievement: 'Congratulations you achieved you physical activity goal of the day'.

- Message 5: You have reached your caloric limit for today. It would be wise to limit your further food intake for today.

Situation options are:

- At home in a hurry

- Cooking dinner

- Having dinner

- Relaxing in front of the television

- Working during a normal day 
- Having a (lunch) break

- Travelling through public transport

- Walking outside

- Doing sports

At the end of this series, the respondents were asked which messages they would like to receive, provided that they would only be sent at appropriate moments during the day. Multiple messages could be selected. Compared to the questionnaire for diabetics, the request to insert a new glucose measurement was replaced by the request to connect their activity monitor to their computer. The medication reminder was replaced by a reminder for taking a break from sitting.

Message options are:

- An overview of your physical activity level of the last week

- Progress towards achieving your physical activity goal of the day

- A request to connect your activity monitor to your computer

- A request to set your physical activity goal

- A message that you have achieved your physical activity goal

- A motivational text (e.g. "You are on the right track")

- Reminder for sitting break: 'You have been sitting for a long time, time for a walk?

- General tips on how to become more physically active

- An explanation of the effects of being physical active on your health

Responses from Office Workers Also for office worker respondents, the most popular situations for receiving different types of messages overall seem to be while relaxing in front of the television, and while having a (lunch) break.

For office users, getting messages while travelling through public transport is also acceptable. The office workers also seemed to be more open to receiving messages while they are at work during a normal day.

Similar to diabetic respondents, a large part of the office users also welcomes motivating and congratulatory messages while walking outside or while doing sports.

Office workers were also hesitant towards receiving messages while at home in a hurry. Receiving messages while cooking dinner was also quite unpopular, except for the message that notified the users that they had already reached their caloric intake limit for that day.

For office workers, there is no unanimity in for what is the best time to send each message. For an overview of their progress towards their daily activity goal and explanations of the effect of physical activity on their health, the four preferred situations seem to be when relaxing in front of the television, when working during a normal day, while trav- elling through public transport or while having a (lunch) break.

They prefer to receive congratulatory messages about their physical activity when they are relaxing in front of the television (!), working during a normal day or while doing sports.

For receiving a message that they have reached their caloric limit for today, the preferred situation is while cooking dinner, while other situations such as while working, having a break or while relaxing in front of the television also received some votes.

When the office worker respondents were asked which messages they would like to receive, provided that the timing was good, two-thirds would like to receive a message containing their physical activity level of the last week, their progress towards their daily physical activity goal, a message that they have achieved their daily activity goal, and a reminder for taking a break from sitting.

Motivational messages or requests to set a new activity goal were welcomed by less than half of the office worker respondents. Less than one-third of the office workers liked to receive requests to connect their activity monitor or general tips on how to become more physically active. Least popular was the explanation of the effects of physical activity on your health, which only one-sixth of the respondents wanted to receive.

\subsection{Adjectives attributed to the system (AttrakDiff)}

At the end of the questionnaire, the respondents were asked to rate the concept that they had seen on different dimensions. This evaluation was based on the AttrakDiff- 2 questionnaire by Hassenzahl [7]. They received a list of 30 word-pairs that fell in one of four scales:

Pragmatic Quality (PQ) Shows product usability, and whether the user can achieve his goals with the product. Hedonic Quality Is divided in two sub-qualities, namely identity and stimulation:

Hedonic Quality—Identity (HQ-I) Indicates how well a user identifies with the product.

Hedonic Quality-Stimulation (HQ-S) Indicates to which extent a product supports the users' need to move forward by offering novel, interesting and stimulating functions, contents, interactions and styles of presentation.

Attractiveness (ATT) Indicates the users' subjective judgement of attractiveness of a product.

Table 1 contains the word pairs in each of the four scales. 


\subsubsection{Diabetics questionnaire}

On average, the diabetic respondents rated the concept on the positive side of the scale for each of the four dimensions, although the values remained close to the neutral line. When these results are split into the different word-pairs, it becomes clear that the respondents were overall rather positive about the attractiveness of the concept, but for the other three dimensions the values fluctuated more (Fig. 10).

The concept scored as rather cautious than bold, which gives it a negative rating on the HQ-S scale. It is also seen as rather technical than human, which lowers the score for the $\mathrm{PQ}$ dimension.

\subsubsection{Office questionnaire}

A similar trend is seen for the average ratings by office worker respondents, where the ratings are on the positive side of the scale, but still rather close to the neutral line.

When looking at the individual word-pairs, it seems that the office workers rated the concept rather positive for both the Pragmatic Quality and Attractiveness, while for Hedonic Quality, the ratings per word-pair fluctuated more (Fig. 11).

When analysing the ratings by gender, it appears that on average female office workers rated the presented concept higher than male office workers.

In the Stimulation-dimension (HQ-S) an especially large difference can be found in the ratings given by male versus female office workers. A more detailed statistic analysis can be found in the next subsection.

When comparing the ratings given by office workers from different age groups, it seems that there are a lot of similarities between respondents in the age groups "Younger than 29" and "Between 30 and 49". Mainly on the Stimulation (HQS) and Attractiveness dimensions, the ratings by respondents aged 50 or older were lower than the ratings by respondents younger than 50 .

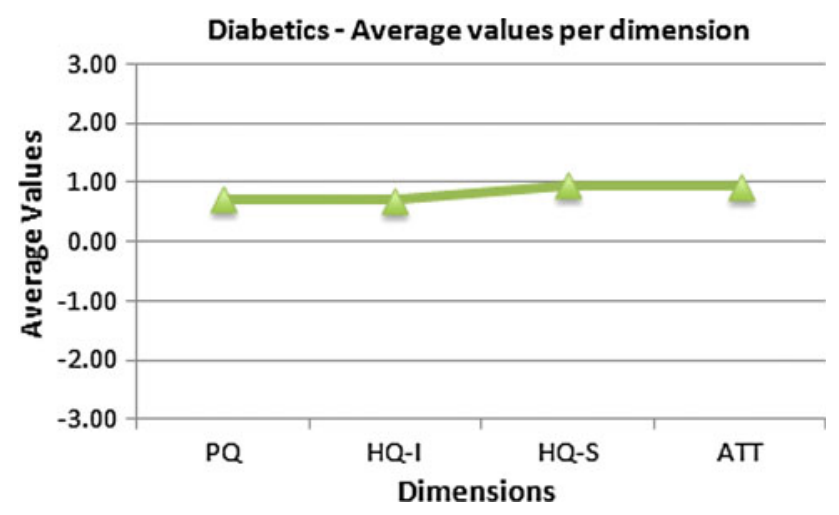

Fig. 10 Average values on scales (diabetics)

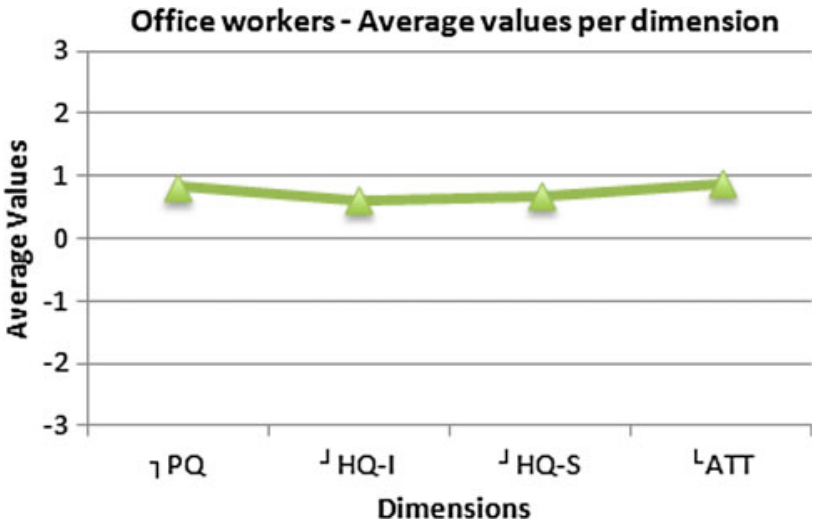

Fig. 11 Average values on scales (office workers)

Figures 12 and 13 show the average scores per item. From these figures it becomes clear that the respondents were overall rather positive about the attractiveness of the concept. In the other three dimensions the values fluctuate more. The concept scores as rather cautious than bold, which gives it a negative rating on the HQ-S scale. It is also seen as more technical than human, which lowers the score for the PQ dimension. When looking at the individual word-pairs, it seems that the office workers rated the concept rather positive for both the Pragmatic Quality and Attractiveness, while for Hedonic Quality, the ratings per word pair fluctuated more.

\subsubsection{Statistics on Attrakdiff results}

Table 1 shows that on the HQ-S scale, among the office workers, females score the concept higher than males on all items. The difference in means for these two groups is significant on the level of 0.05 on all but one item (cautious-bold). The table also shows that the HQ-S scale differs in this respect from other scales. Moreover, we do not see such a systematic and significant difference between females and males within the group of diabetic patients. Notice that on all seven ATT items among the Office Workers females score higher than males where among the diabetes females score lower than males on all the ATT items. But here differences are only significant on one item and only for office workers.

In assessing these results we should also look at the reliability scores for the different scales. Table 2 shows the Cronbach alpha values for the whole group and for the two user groups separately. We see that for office workers the reliability is good $(>0.7)$ for all four scales and also higher than for the diabetes group. For the latter group only on the ATT scale the reliability score is good enough. In particular, the HQ-S scale shows on the office workers data a high Cronbach alpha value (0.891). This gives some support for the conclusion that female and male office workers differ in their assessment of the system on the HQ-S scale. 
Fig. 12 Average values on scales per item (diabetic patients)

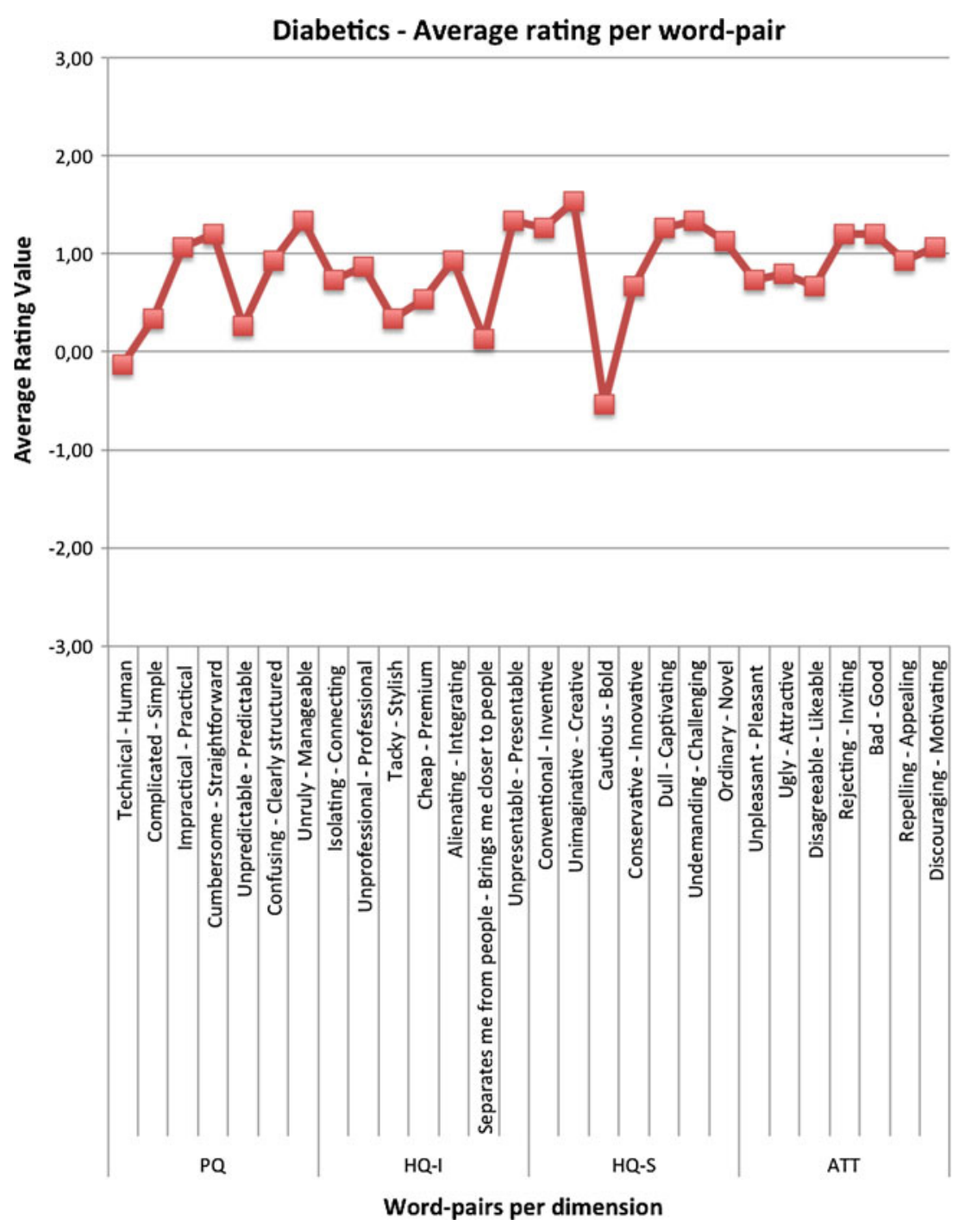

3.5 Remarks made after filling in the questionnaire

After answering all questions, users had the opportunity to make remarks about the presented concept or about the test itself.

\subsubsection{Remarks from diabetics}

"In NL insurance does not pay for glucose monitoring if your diabetes is well managed. In the US insurance doesn't always pay for such either, but more often than in NL. Also realize that many people don't take medication, like me, I haven't for over a year now. I'm also interested to know if you've considered continuous glucose monitoring. I know it can be invasive, and generally the few people who do have it are Type I, but it can be a good tool to get a lot of data to really help a person."
During previous interviews with diabetics, this issue also came up already. Not all diabetic patients take medication, and not all of them have a glucose monitor. This is why they application should be highly adaptable to the users' personal situations.

"Tests also in other languages e.g. Dutch For those who can not read English."

We noticed that there were very few respondents to our questionnaire for diabetics, which was first only available in English. Therefore, we also created a Dutch version.

\subsubsection{Remarks from office workers}

'I'm not an average person - I'm the type that hates navigating systems in cars... But, having said that, the idea of a light that could show its approval or disapproval of my habits is rather inviting!" 
Fig. 13 Average values on scales per item (office workers)

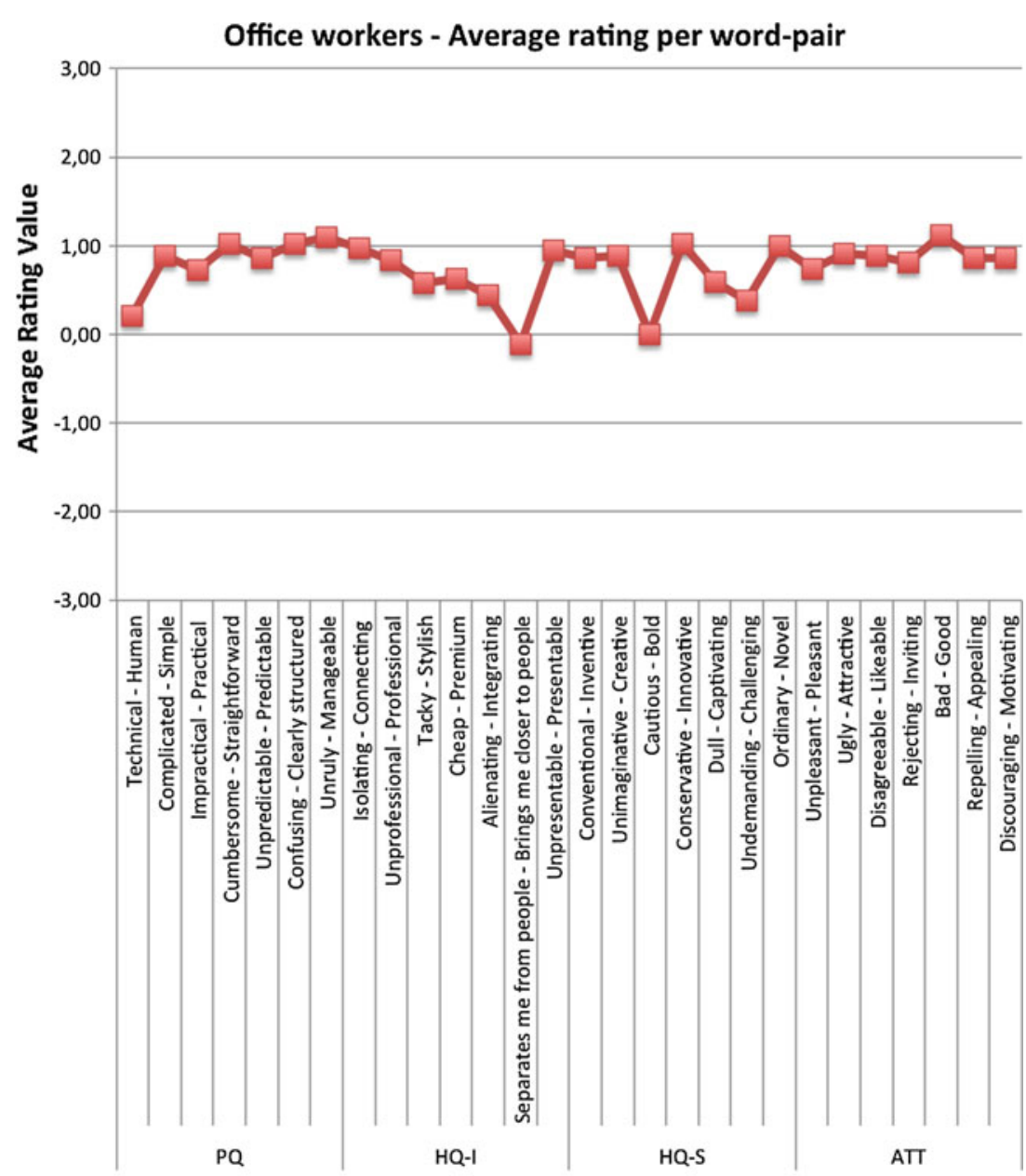

Word-pairs per dimension
Table 2 AttrakDiff Scales Reliability Analysis (Cronbach alpha values)

\begin{tabular}{llll}
\hline Scale & All $(N=58)$ & Office $(N=43)$ & Diab. $(N=15)$ \\
\hline PQ & 0.666 & 0.711 & 0.573 \\
HQ-I & 0.692 & 0.765 & 0.334 \\
HQ-S & 0.820 & 0.891 & 0.412 \\
ATT & 0.846 & 0.893 & 0.700 \\
\hline
\end{tabular}

Although very little of the respondents actually owned a lamp that could change colors, from the preferences it became clear that many respondents would actually prefer to receive feedback through an ambient device such as the color-changing lamp.

"There has to be a larger difference to current available systems. The advice from these systems has never triggered me yet to start doing something and the ideas are not imaginative at all". By extending the services to multiple devices, Smarcos aims at bringing a more context-aware, personalized service.
"I would want very much control over when she can send me what messages" "The concept is interesting. The balance between motivating the user and annoying them through nagging needs to be carefully considered."

Personalization of which messages users want to receive and limitations on when to send messages are very important for the success of the application.

\section{User evaluations with the Smarcos system}

Results of the online questionnaires were used to finish the first prototype of the Smarcos system. This prototype was evaluated in two small user evaluations with both target groups, one diary study of 1 week with five diabetes type II patients and one user evaluation of 1 week with nine office workers. The diabetes type II patients used the system on their computer and smartphone and receive feedback about medication intake and physical activity. Office workers used the system on their smartphone and received feedback about their physical activity. During the evaluation of the system with office workers we were interested in the effect of present- 
Table $1 P$-values from independent t-test for differences in means per item between females and males for all users $(n=58, f=31, m=27)$, and split for office workers $(n=43, f=20, m=23)$ and diabetic $(n=15, f=11, m=4)$

\begin{tabular}{|c|c|c|c|c|}
\hline AttrakDiff scale & Semantic pairs & All $(N=58)$ & Office workers $(N=43)$ & Diabetics $(N=15)$ \\
\hline \multirow[t]{7}{*}{ PQ } & Technical-Human & & & \\
\hline & Complicated-Simple & & & \\
\hline & Impractical-Practical & & & \\
\hline & Cumbersome Straightforward & & & \\
\hline & Unpredictable Predictable & $0.000(F<M)$ & $0.006(F<M)$ & $0.048(F<M)$ \\
\hline & Confusing-Clearly structured & & & \\
\hline & Unruly-Manageable & & & \\
\hline \multirow[t]{7}{*}{ HQ-I } & Isolating-Connecting & & & \\
\hline & Unprofessional-Professional & & & \\
\hline & Tacky-Stylish & & & \\
\hline & Cheap-Premium & $0.019(F>M)$ & $0.008(F>M)$ & \\
\hline & Alienating-Integrating & $0.011(F>M)$ & $0.019(F>M)$ & \\
\hline & Separates-Closer to people & & & \\
\hline & Unpresentable-Presentable & & & \\
\hline \multirow[t]{7}{*}{ HQ-S } & Conventional-Inventive & $0.017(F>M)$ & $0.001(F>M)$ & $F>M$ \\
\hline & Unimaginative-Creative & $0.020(F>M)$ & $0.044(F>M)$ & $F<M$ \\
\hline & Cautious-Bold & $F>M$ & $F>M$ & $F<M$ \\
\hline & Conservative-Innovative & $0.011(F>M)$ & $0.001(F>M)$ & $F>M$ \\
\hline & Dull-Captivating & $0.017(F>M)$ & $0.042(F>M)$ & $F>M$ \\
\hline & Undemanding-Challenging & $0.005(F>M)$ & $0.042(F>M)$ & $F>M$ \\
\hline & Ordinary-Novel & $0.011(F>M)$ & $0.002(F>M)$ & $F<M$ \\
\hline \multirow[t]{7}{*}{ ATT } & Unpleasant-Pleasant & & $F>M$ & $F<M$ \\
\hline & Ugly-Attractive & & $F>M$ & $F<M$ \\
\hline & Disagreeable-Likeable & & $F>M$ & $F<M$ \\
\hline & Rejecting-Inviting & & $F>M$ & $F<M$ \\
\hline & Bad-Good & & $F>M$ & $F<M$ \\
\hline & Repelling-Appealing & & $F>M$ & $F<M$ \\
\hline & Discouraging-Motivating & & $0.019(F>M)$ & $F<M$ \\
\hline
\end{tabular}

Empty entries: $p>0.05 . F>M$ indicates that females score higher than males

If no p-value is given the difference is not significant on the 0.05 level

ing feedback using different output modalities. One group of office workers received Feedback about their physical activty as a text messages while the other group of office workers received feedback presented by an embodied conversational agent (ECA). In this section we present the results of these two user evaluations.

\subsection{Diary study with diabetes patients}

The goal of this diary study was to investigate if the expected use of the system matches the actual use of the system by the participants in their daily lives.

During the diary test we want to investigate:

1. The possible problems with the conceptual and navigation model of the system. Do the users understand the icons, metaphors and overall design? And, are the users able to find their way through the application efficiently and can they access the most frequently used functions easily?

2. Whether the functionality and work flow of the system meet the expectations of the user. Does the system support the tasks most frequently performed by the user? Do users know that they can migrate to other devices and do they have the feeling that information states are synchronized across the devices?

3. Whether the system is easy enough to learn. We wanted to see if the system is easy to learn by the users in their own environment and we wanted to measure the attractiveness of the user interface.

4. The users' opinion regarding the coaching provided by the system. Do user except the way of getting feedback 
Fig. 14 Smarcos diabetes app on a computer

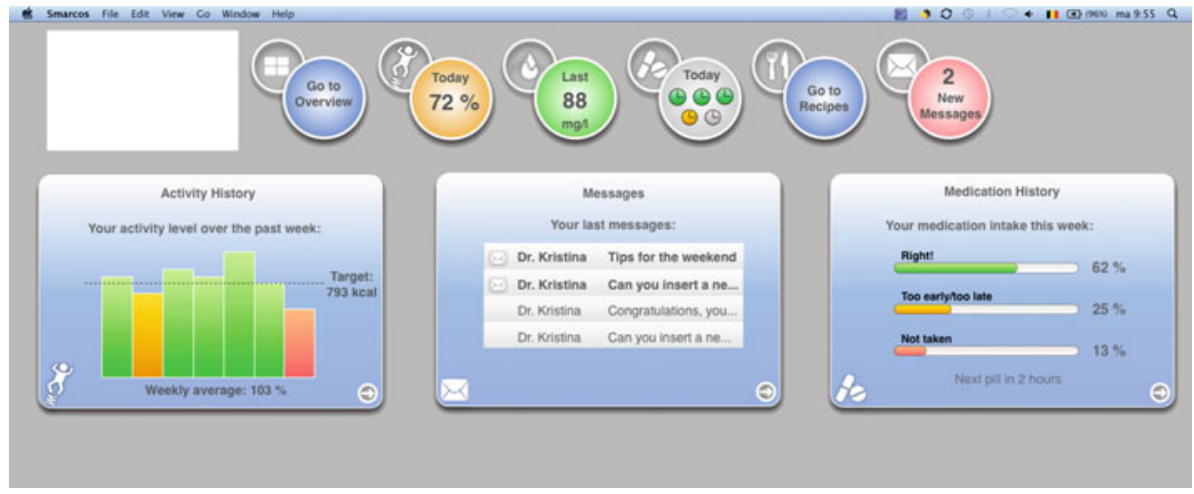

and support, in what are the situations users want to get feedback or reminders and do users trust the feedback and suggestions from the system? What is the perceived effectiveness of the system. And what are the privacy issues involved in using such a system.

The next section will present the diary study in more detail.

\subsubsection{Method}

Five diabetes type II patients worked with the Smarcos system for 1 week in their own home and work environment. The participants were using the application on their own computer or laptop (see Fig. 14) and smartphone (see Fig. 15) (iPhone or Android) including a activity monitor and smart pill dispenser. The diary evaluation were performed in June 2012 and July 2012. At the beginning of the test, each participant was visited at his/her home, where (s)he received the devices and applications needed to execute the prototype test. Each introductory visit took about 1.5 hour. After the introduction, the participants used the Smarcos system including the different devices for a whole week. During this week, they were asked to regularly upload their activity data. The Smarcos system provided feedback about to the users about their activity level and their medication intake. Furthermore, the users received messages from their virtual coach. Each day, each participant filled out a diary with questions. The aim of these questions was to gather information about the users' interactions with the system that cannot be gathered from the database logs. The AttrakDiff questionnaire was part of the diary as well. During the evaluation log data about docking moments, medication intake and messages were stored. The diary contained contact information in case of problems, questions and remarks. Shortly after the test week an evaluation visit took place with each of the participants. During this visit all the devices were collected and the users experiences were discussed. The information collected by the participants in their diaries served as basis for these discussions.

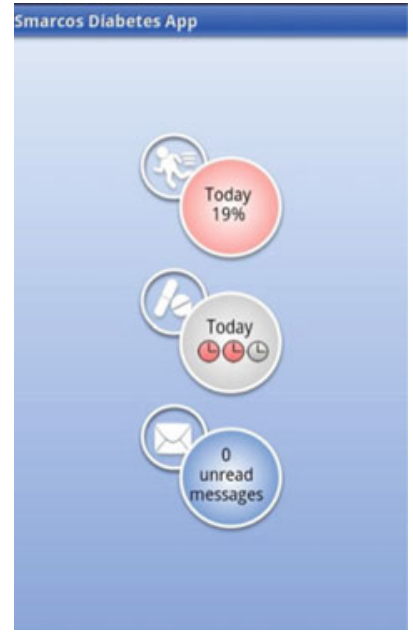

Fig. 15 Smarcos diabetes app on a smartphone

Table 3 Demographics of the participants in the diary study

\begin{tabular}{llll}
\hline Participant & Gender & Age & Diagnosed since \\
\hline 1 & Man & 30 & 2 years \\
2 & Man & 40 & 2 years \\
3 & Woman & 52 & 2 years \\
4 & Woman & 43 & 1.5 years \\
5 & Woman & 43 & 1.5 years \\
\hline
\end{tabular}

\subsubsection{Results}

This section will discuss the results of the diary study.

Demographics Five users participated in the diary study, two males and three females. Table 3 shows more details about the participants. All participants use a computer for work or in private life. Four participants own a smartphone, one did not have a smartphone and received a smartphone for the diary evaluation.

Interaction with the system During the evaluation data about docking moments, messages, medication intake and interaction with the system were logged. Table 4 shows aver- 
Table 4 Interaction with the system during diary study

\begin{tabular}{lc}
\hline Type of interaction & \# Interactions \\
\hline Uploading activity data & 9.2 \\
Opening pill dispenser & 20.2 \\
Messages medication reminder & 3.4 \\
Messages low activity & 0.2 \\
Messages 100 \% medication score & 1 \\
Messages dock request & 0.4 \\
Medication overview on smartphone & 12 \\
Medication overview on computer & 7.8 \\
Activity level on smartphone & 7.6 \\
Activity level on computer & 6.8 \\
\hline
\end{tabular}

ages of interaction with the system per participant over the week.

Diary and interview results During the test participant filled in the diary and after the test, the participants were interviewed. The most important outcomes of those interviews and diaries are presented in this section.

Characteristics of the system The participants indicated that they found the activity monitor: innovative, motivating, challenging, convenient. Most of them experienced the docking of the activity monitor as: easy to handle, convenient. One of the participants indicated that she found this complicated (she did not succeed to dock during the test).

Participants indicated that found the smartphone application: presentable, nice, clearly structured, convenient.

Some of the participants found the medication dispenser: convenient, easy to handle. Others indicated that they found it confusing (because of the structured intake schedule that was set, in daily life the intake moments vary more), difficult to handle (because of the size).

The participants were all positive about the messages, indicating that they found these: motivating, structured.

General impression During the interviews all the participant agreed that the system did not take much time to work with and was easy to use. Only docking the activity monitor took some time and effort even though users just needed to connect it through a USB connector. Here are some of the most important answers the participants gave during the test (translated from Dutch).

Results from the diaries and interviews showed that user were able to use the system.

"You don't notice much of the system if you think of your medication yourself."

"Super easy, the system is straightforward."

"The system speaks for it self and was clear."

"The system was not difficult to use. When I didn't understand something my husband was able to help me."
The user interface of the application was clear and users used the applications on both devices. User had the impression that the different applications were synchronized.

"I like the application on the computer because of the bigger screen."

"The application on the computer did not add much because everything was also on my smartphone."

"It was nice to have multiple devices. I am not a real technical person, but for me it was clear and easy to use."

The system was easy and quick to learn.

"Learning the system and using the applications did not cost much time."

The participants found especially the activity monitor interesting and found it motivating to be able to see which level of activity they really reach.

"Receiving messages about the $100 \%$ score on medication intakes were nice and motivating. Receiving messages are not annoying if you know that the system is right."

"The system creates awareness. You do not realize what you do in daily life. This makes you reflects your own behaviour."

The participants were positive about using the system.

"Only if I would forget my medication, but that is not the case."

"The activity monitor is interesting, I compare this to seeing how much fuel your car uses."

"It would be nice to have insight in my activity during my daily life, because I want to be more active."

"Absolutely!"

"I know that I'm an active person and I'm really precise with my medication. I don't think such a system is useful for me."

Participants think that a system like this can help people to get a healthier lifestyle.

"It is stimulating to see how active I was during the day. I want to reach the $100 \%$ target, but I don't know if it works like that for everyone."

"Receiving feedback is stimulating for people."

"The system gives you insights which can be very motivating, since people often don't know what the situation is. It will get them thinking."

"Especially the activity monitor will make people aware. Normally you are not that aware of your activity."

AttrakDiff Figure 16 shows the average values in the four dimensions of the AttrakDiff questionnaire [7] for the prototype evaluation of the Smarcos system. The scores per word pair can be found in Fig. 17. On average, the participants rated the concept on the positive side of the scale for 


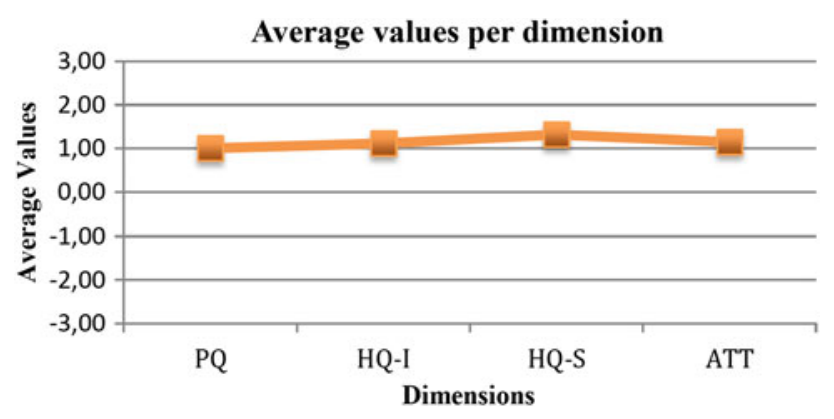

Fig. 16 Average values on scales for the diary evaluation

each of the four dimensions, although the values remained close to the neutral line. The users rated the prototype as simple, clearly structured, stylish, inventive, innovative and motivating.

We did not look into a statistical analysis of the AttrakDiff results because of the small number of participants.

4.2 User evaluation with an embodied conversational agent

In a two week user evaluation we evaluated the physical activity coaching system for office workers on smartphones (see Fig. 18). In this user evaluation we compared two different

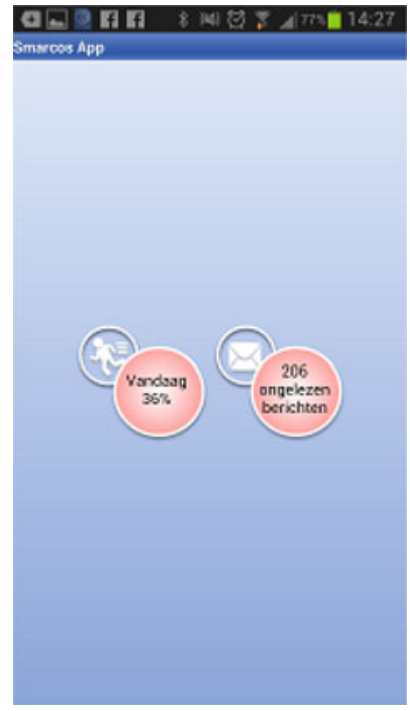

Fig. 18 Physical activity coaching application on a smartphone

ways of presenting the feedback to the user. One version presented the feedback as a simple text message (see Fig. 19). The other version of the system presented the feedback by an ECA (see Fig. 20). The ECA showed facial expression while speaking out the feedback. To present the feedback
Fig. 17 Average values on scale per item for the diary study
Average rating per word-pair

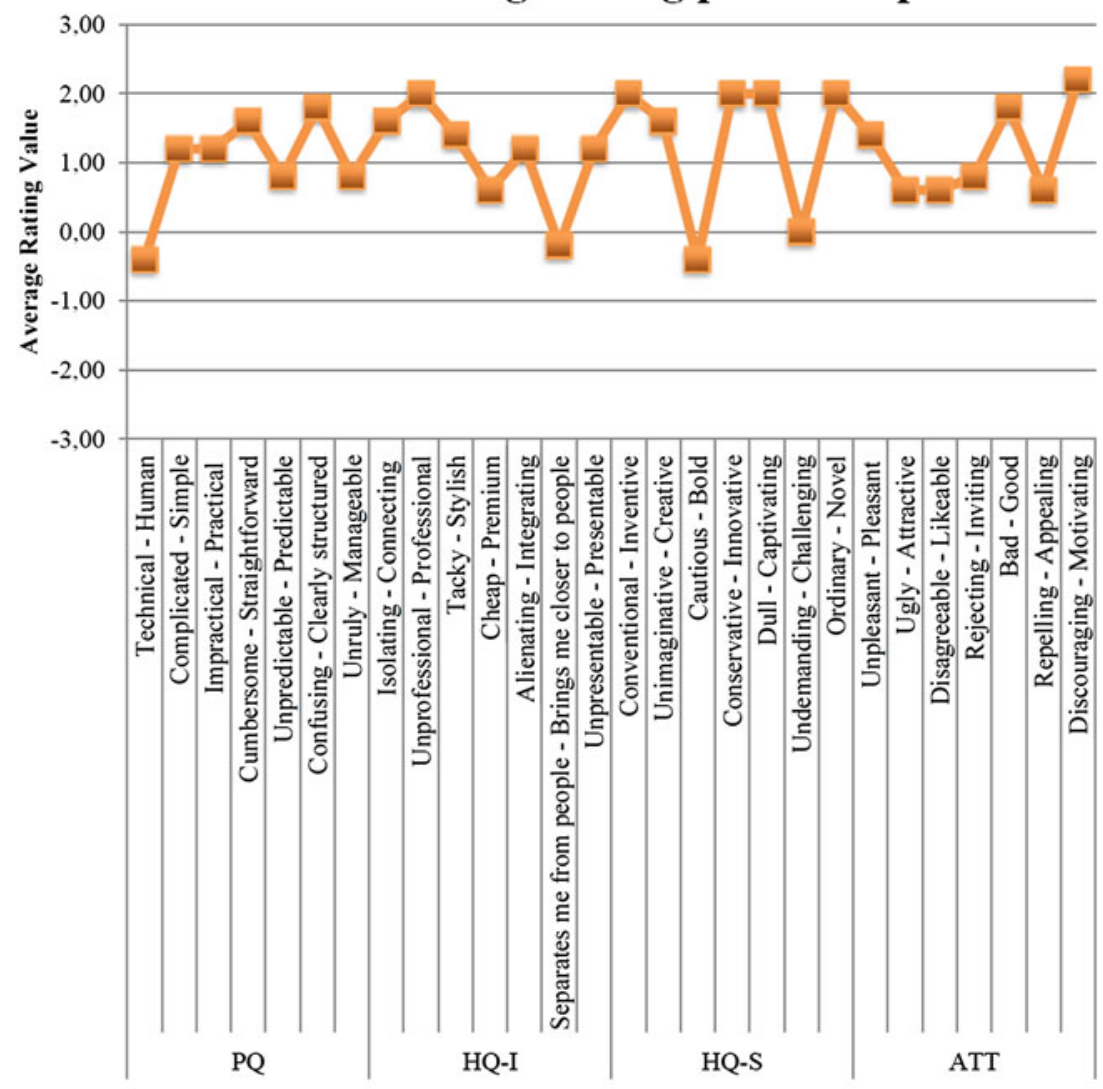

Word-pairs per dimension 
by an ECA we used the Elckerlyc system for Android ${ }^{3}$ [8]. Using an ECA for presenting feedback in a coaching system can have positive effects on the user experience and the effectiveness of the coaching program $[1,2]$. The goal of this evaluation to investigate if the system offers what users expect from a physical coaching system and if there were differences in user experience between the two ways of presenting feedback.

\subsubsection{Method}

Nine office workers participated in the user evaluation. The users used only the Smarcos applications on their smartphone. To measure physical activity the activity monitor was used. At the beginning of the evaluations users got an introduction to the evaluation and received the activity monitor. The first week was an assessment week. During this week the user had to carry the activity monitor during the day to measure a baseline and received no feedback. After the assessment week every user received his or her unique activity goal for the next week and the Smarcos applications. In the second week users received feedback about their progress and reminders and tips to be more physical active and to connect the activity monitor daily. During the second week users were invited to fill in several online questionnaires $[4,7,10,18]$ about the experiences with the system. The AttrakDiff questionnaire was used to measure user experience. After the second week all users were visited to discuss their experiences with the system. The results of the questionnaires act as a basis for this interview. During the user evaluation log data about the interactions with the system were stored.

\subsubsection{Results}

This section presents the first results of the user evaluation of the Smarcos physical activity coaching system.

Demographics Nine users participated in the diary study, four males and five females. All participants use a computer for work or in private life. Eight participants own a smartphone, one did not have a smartphone and received a smartphone for the diary evaluation. The participants sit $7.3 \mathrm{~h}$ on an average working day.

General impression of presentation of feedback The participants were able to use the Smarcos applications and it did not take much time the use the system, read messages of connect the activity monitor. The participants of the user evaluation were asked for their opinion about the way the feedback was presented. Users stated that feedback presented by an ECA can be more motivating: "When a person or a face is talking to me, it feels more personal addressed to me"

\footnotetext{
${ }_{3}^{3}$ For more information about Elckerlyc on Android see http://youtu. be/Ow-EDHqRgvU.
}

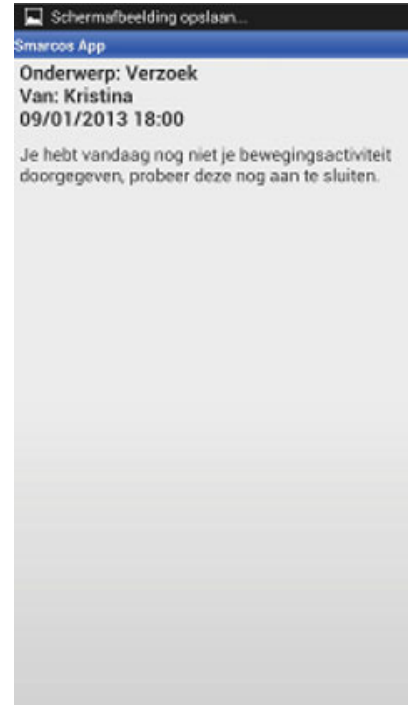

Fig. 19 Text message on a smartphone

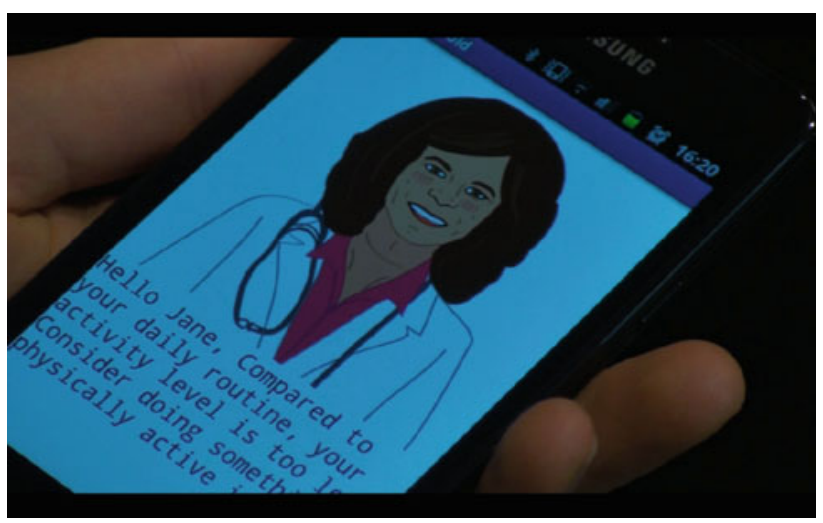

Fig. 20 ECA message on a smartphone

and "I think an ECA can be more motivating. It is difficult to explain but I could imagine an ECA is more motivating than a text message". Other participants stated that a text message will be a better way of presenting the feedback: "I prefer text messages. No, I do not feel anything for the ECA, it look childish to me" and "The ECA will be annoying. It reminds me to Clippy of MS Word.". Participants stated that a physical activity coaching program could help to live a healthier life, but the user should be open to such a system and react to the feedback (by being more physical active) and change his behaviour "This system will only work for people how want to use it. If you do not want to have such a system, you simple ignore the messages and continue with you daily life".

"If you really want to use the system it can help you to live a healthier life, but you have to be open to use the system. Only than it will work. It works better than using a step counter." Participants liked to receive feedback when they reached their goal during the day.

AttrakDiff The scores per word pair of the AttrackDiff questionnaire [7] for the evaluation of the Smarcos system 
Fig. 21 Average values on scale per item for the physical activity coach for office workers

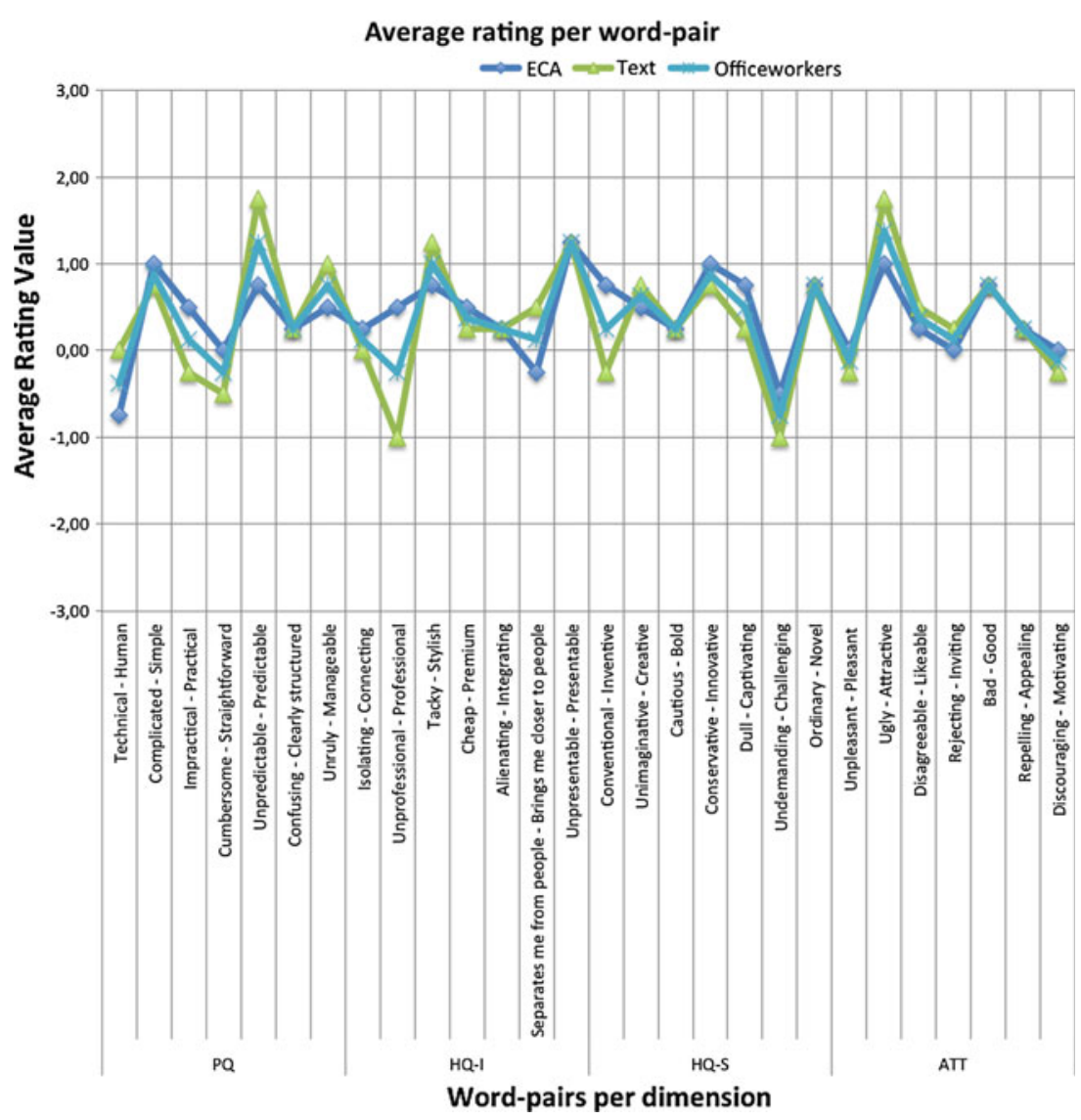

can be found in Fig. 21. It shows the results of the complete system and as well as the separate results of the text and ECA coach.

On average the office workers rated the physical activity coaching system neutral. Users rated the system undemanding rather than challenging, predictable rather than unpredictable and attractive rather than ugly. The differences between the system with the ECA and the system with the text only feedback are small. Users rated the ECA feedback as more professional.

\subsection{Conclusion}

The results of the two small user evaluations show that the Smarcos coaching system was easy to use by both target groups. The diabetes type II patients were positive about the system and think such a system can help and motivate people to live a healthier life. Office workers showed different opinions about the presentation of feedback. Participants stated that a system like this could help the user to live a healthier life, but users should be open to a coaching system. Some participants stated that feedback presented by an ECA can be more motivating. Other participants stated that they did not like an ECA for presenting feedback and that they preferred a text message. Looking at the results of the AttrakDiff questionnaire we see that the ECA feedback is rated as more professional than text feedback.

Figure 22 combines the outcomes of the AttrakDiff questionnaires of the diary evaluations with the result of the AttrakDiff questionnaire from the online user evaluation with diabetes type II patients. When we look at the scores per item we can see a difference between the online prototype evaluation and the diary evaluations with the Smarcos system. The diabetes type II patients in the diary evalutions with the Smarcos system rated the system more undemanding compared to the online prototype evaluation where participants rated the system more challenging. User of the prototype system rated the system as more professional compared to the results from the online evaluation of the system.

Figure 23 shows the combined outcomes of the AttrakDiff questionnaires of the user evaluation and the online evaluation. Office workers in the user evaluation rated the system as less motivating, more cumbersome and less professional than office workers in the online evaluation. Office workers rated the ECA feedback as more professional than the text feedback.

The timing of the feedback messages during the user evaluations were based on time and event triggers. Time triggered messages are sent on fixed moments during the day (e.g. every day at 18:00 or on days when the user did not con- 
Fig. 22 Combined results of the AttrakDiff questionnaires for diabetes type II patients

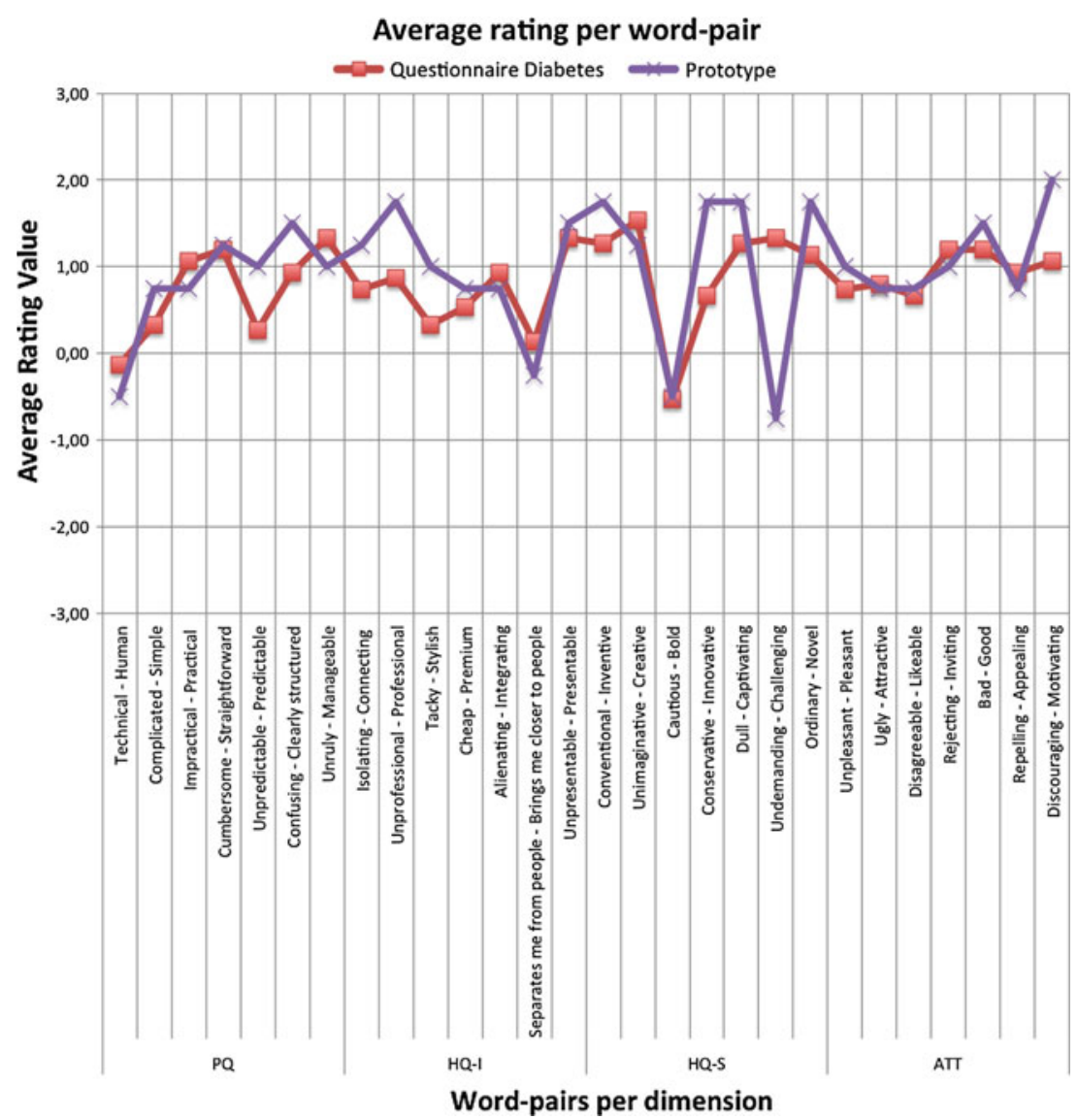

nect his activity monitor). Event based messages are sent when the user connects his activity monitor. In the follow up version of the coaching system we make use of context triggers for presenting feedback. Our study shows that users prefer receiving feedback messages during lunch time or in the evening when they return home. To be able to recognize these event we will make use of the LifeLiner of VTT. ${ }^{4}$ The Lifeliner mobile application runs in the background of a smartphone and automatically detects the patterns of daily routines. Special events, like "lunch" and "returning home" can be specified and detected.

Diabetes type II patients were positive about the system and stated the it will help to live a healthier life, office workers were (less) positive about the system and stated that a system like this only will help to live a healthier life if the user is open to use the system and really wants to change his behaviour. How can the diverging opinions between these two user groups be understood? A tentative argument might be that diabetes type II patients used the system while coping their disease. They had to take their medication at several times. So there was a real need to use the system. The office workers were just asked to join the user evaluation. There

\footnotetext{
${ }^{4}$ More information about LifeLiner can be found on http://cauit.erve. vtt.fi/lifeliner/ and http://youtu.be/xT4Cg8Y48Pg.
}

was no pressure and actual need to change their behaviour and to be more physical active. Further research is needed to draw more definite conclusions about the relation between user assessments of this type of systems and the motivations users have in using the system.

\section{Conclusion and future work}

Users have a preference for receiving feedback on the smartphone, computer, or television, although there also seems to be some interest in getting feedback through the colorchanging lamp. Situations that users would mainly like to receive feedback in, is while relaxing in front of the television or while having a (lunch) break. As for the type of messages that can be sent, users seem to have a preference for reports about their progress, rather than advice or learning messages. It also appears that respondents are rather hesitant towards receiving messages. Most messages were only welcomed by half of the respondents or less. Therefore, careful personalization and limitations on when to send messages are a necessity.

According to the AttrakDiff ratings, users are positive about the presented concept, but there is room for improvement. 
Fig. 23 Combined results of the AttrakDiff questionnaires for office workers

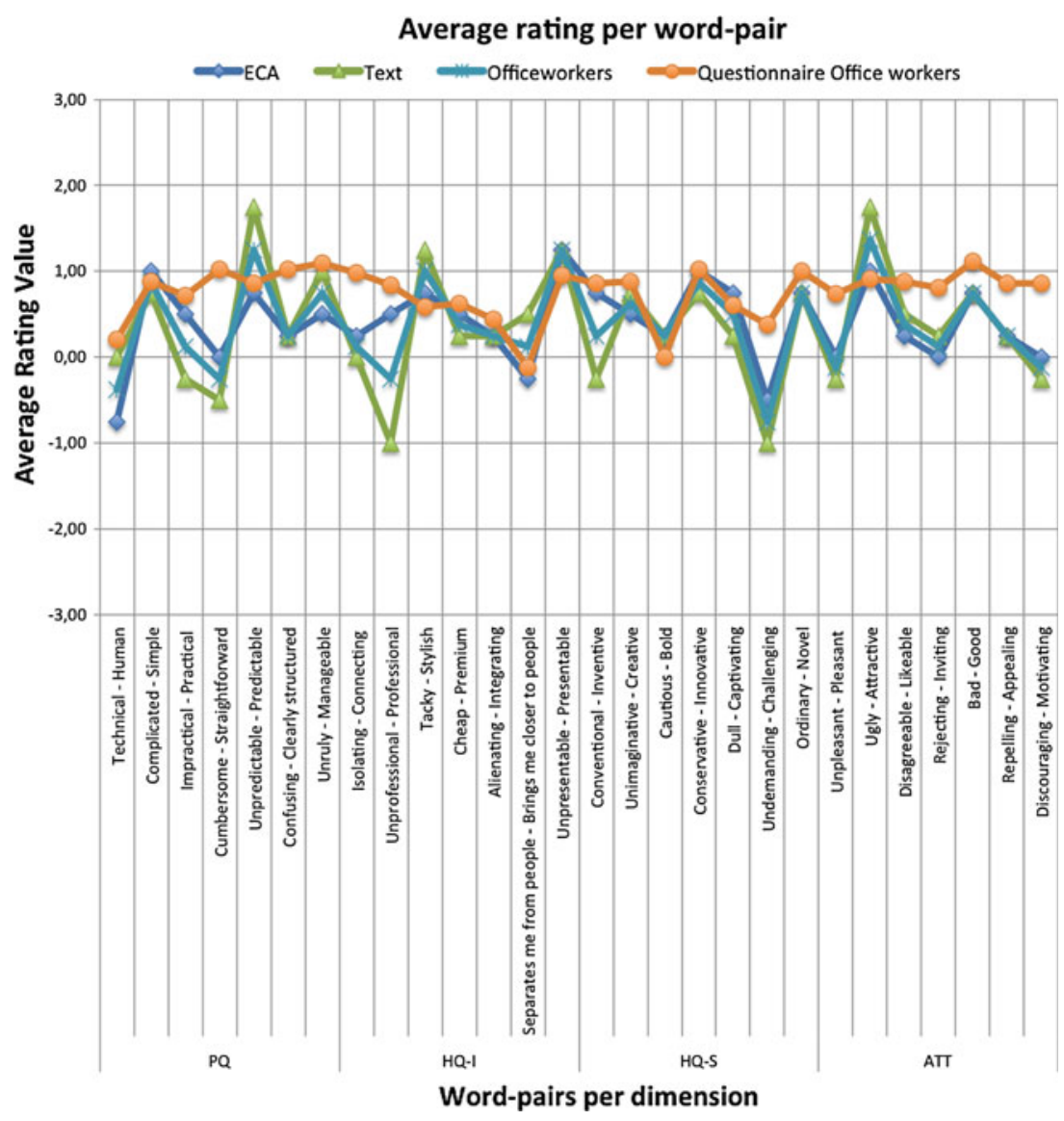

It would be advisable to further research in which situations it is OK to send which messages on which device, as we only presented a very limited amount of situations, messages, and devices in our questionnaire.

Finally, quite a few ratings we measured in the Attrakdiff evaluation are relatively close to the neutral line. Neutral ratings can mean either "I don't know what these terms mean", "I don't have an opinion on this" or "I think the concept scores somewhere in the middle between these two terms". The fact that the ratings are based on a description of the concept and not on the actual use of the concept, might have an influence on this.

Overall the responses of the users during the evaluations of the prototype were positive. Results of the user evaluations with the Smarcos system showed that user were able to learn and use the system easily. Diabetes type II patients were positive about the system and think a coaching system like this can help people to live a healthier life. Office workers stated that the system could help people to live a healthier life, but the user should be open to system to really use it. If you do not want to use the system you simple ignore the messages and continue with your daily activities. The participants had mixed feelings about the presentation of feedback by text or an ECA. They could imagine that feedback presented by an
ECA can be more motivating, but some stated also that they prefer text feedback above ECA feedback. The participants thought that the ECA will be childish and annoying over time.

Results of new user evaluations where we compare the ECA feedback with the text feedback, over a longer period of time including more participants, will provide more understanding about the effects on the user experience and effectiveness of the coaching program. The integration of LifeLiner into the Smarcos coaching system will result in a system that is able to use context triggers for the timing of feedback messages. Users will receive feedback at their actual lunch time, or when they return home after work.

Another topic of interest is to extend the coaching system with a dialog system. At this moment the interaction with the system is one direction. The system sends feedback to the user or the user uses the system to look for some detailed information. Adding the opportunity of having a dialogue with the system will improve the interaction with the system. User can for example ask for more details about a specific feedback message or the system can use question answer dialogues to get more information from the user about their physical, medication or food activities. The interaction with the system can be based on text-to-speech and voice recognition including the ECA or can be text based using text and but- 
tons in the user interface. An early prototype of such a system was developed, but future development is needed to test it in a user evaluation.

Acknowledgments The authors are grateful to all participants that have filled-in the survey, participated in the user evaluations and to all Smarcos partners who were involved in the research reported in this paper.

Open Access This article is distributed under the terms of the Creative Commons Attribution License which permits any use, distribution, and reproduction in any medium, provided the original author(s) and the source are credited.

\section{References}

1. Bickmore T, Mauer D, Crespo F, Brown T (2007) Persuasion, task interruption and health regimen adherence. In: Proceedings of the 2nd international conference on Persuasive technology, PERSUASIVE'07. Springer, Berlin, pp 1-11

2. Blanson Henkemans OA, van der Boog PJM, Lindenberg J, van der Mast CAPG, Neerincx MA, Zwetsloot-Schonk BJHM (2009) An online lifestyle diary with a persuasive computer assistant providing feedback on self-management. Technol Health Care 17(3): 253-267

3. Consolvo S, McDonald DW, Landay JA (2009) Theory-driven design strategies for technologies that support behavior change in everyday life. In: Proceedings of the 27th international conference on Human factors in computing systems, CHI '09. ACM, New York, pp 405-414

4. Cote J, Yardley J, Hay J, Sedgwick W, Baker JR (1999) An exploratory examination of the coaching behaviour scale for sport. AVANTE 5:82-92 (ISSN=1201-6144)

5. Cramer JA (2004) A systematic review of adherence with medications for diabetes. Diabetes Care 27(5):1218-1224

6. Geleijnse G, Van Halteren A, Diekhoff J (2011) Towards a mobile application to create sedentary awareness. In: Eslambolchilar P, Wilson ML, Oakley I, Dey A (eds) Proceedings of the 2nd international workshop on persuasion, influence, nudge and coercion through mobile devices (PINC2011), vol 722, pp 90-111

7. Hassenzahl M (2004) The thing and I: understanding the relationship between user and product. Kluwer, Norwell, pp 31-42

8. Klaassen R, Hendrix J, Reidsma D, op den Akker HJA (2012) Elckerlyc goes mobile: enabling technology for ecas in mobile applications. In: The sixth international conference on mobile ubiquitous computing, systems, services and technologies, Barcelona, Spain. XPS (Xpert Publishing Services), pp 41-47
9. Klaassen R, op den Akker HJA (2012) Inter-usability and the presentation of multi-modal feedback for physical activity and diabetic type ii patients. In: Proceedings of measuring behavior 2012, 8th international conference on methods and techniques in behavioral research, Wageningen. Noldus Information Technology, pp 115117 (ISBN=978-90-74821-87-2)

10. McCroskey JC (1966) Scales for the measurement of ethos. Speech Monogr 33:65-72

11. Mukhtar H, Belaïd D, Bernard G (2011) Dynamic user task composition based on user preferences. ACM Trans Auton Adapt Syst 6(1):4:1-4:17

12. Noar SM, Benac CN, Harris MS (2007) Does tailoring matter? Meta-analytic review of tailored print health behavior change interventions. Psychol Bull 133(4):673-693

13. Oinas-Kukkonen H (2010) Behavior change support systems: a research model and agenda. In: Oinas-Kukkonen H, Ploug T, Hasle P (eds) PERSUASIVE (2010). LNCS, vol 6137. Springer, Berlin, pp 4-14

14. op den Akker H, Hermens HJ, Jones VM (2011) A context-aware adaptive feedback system for activity monitoring. In: 2nd international conference on ambulatory monitoring of physical activity and movement, ICAMPAM 2011, Glasgow, United Kingdom, vol 2. ICAMPAM, Glasgow, pp 16.2

15. op den Akker R, Klaassen R, Lavrysen T, GeleijnseG, van Halteren A, Schwietert H, van der Hout M (2011) A personal context-aware multi-device coaching service that supports a healthy lifestyle. In: Proceedings of the 25th BCS conference on human-computer interaction, BCS-HCI '11. British Computer Society, Swinton, pp 443448

16. Prochaska JO, Velicer WF, Rossi JS, Goldstein MG, Marcus BH, Rakowski W, Fiore C, Harlow LL, Redding CA, Rosenbloom D, Rossi SR (1994) Stages of change and decisional balance for twelve problem behaviors. Health Psychol 13:39-46

17. Visser FS, Stappers PJ, van der Lugt R, Sanders E (2005) Contextmapping: experiences from practice. CoDesign 1(2):119-149

18. Venkatesh V, Morris MG, Davis GB, Davis FD (2003) User acceptance of information technology: toward a unified view. MIS Q 27(3):425-478

19. Vervloet M, van Dijk L, Santen-Reestman J, van Vlijmen B, Bouvy M, de Bakker D (2011) Improving medication adherence in diabetes type 2 patients through real time medication monitoring: a randomised controlled trial to evaluate the effect of monitoring patients' medication use combined with short message service (sms) reminders. BMC Health Serv Res 11(1):5 